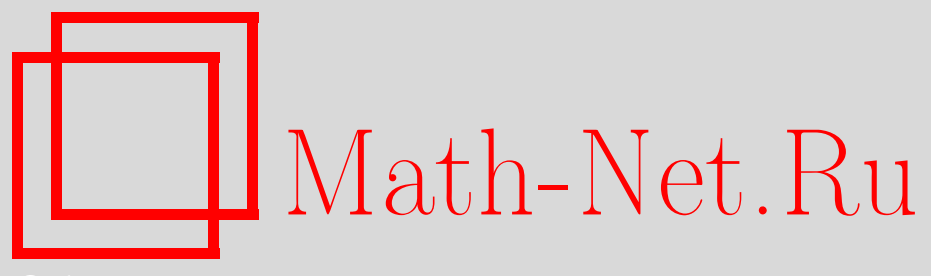

В. И. Иванов, А. В. Иванов, Оптимальные аргументы в неравенстве Джексона-Стечкина в $L_{2}\left(\mathbb{R}^{d}\right)$ с весом Данкля, Матем. заметки, 2014, том 96, выпуск 5, 674686

DOI: https://doi.org/10.4213/mzm10535

Использование Общероссийского математического портала Math-Net.Ru подразумевает, что вы прочитали и согласны с пользовательским соглашением http://www . mathnet.ru/rus/agreement

Параметры загрузки:

IP : 54.198 .64 .247

26 апреля 2023 г., 18:05:55

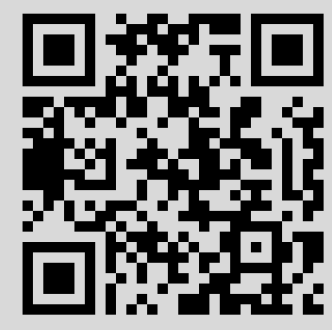




\section{Математические заметки}

Том 96 выпуск 5 ноябрь 2014

УДК 517.5

\section{Оптимальные аргументы}

в неравенстве Джексона-Стечкина в $L_{2}\left(\mathbb{R}^{d}\right)$ с весом Данкля

\section{В. И. Иванов, А. В. Иванов}

Работа посвящена исследованию оптимальных аргументов в точном неравенстве Джексона-Стечкина с модулем непрерывности порядка $r$ в пространстве $L_{2}\left(\mathbb{R}^{d}\right)$ с весом Данкля, определяемого системой корней $R$ и неотрицательной функцией кратности $k$. Если

$$
\lambda_{k}=\frac{d}{2}-1+\sum_{\alpha \in R_{+}} k(\alpha)=\frac{1}{2},
$$

где $R_{+}$- положительная подсистема системы корней, то оптимальные аргументы для всех $r$ совпадают. Если $\lambda_{k} \neq 1 / 2$, то оптимальный аргумент для модуля непрерывности второго порядка больше, чем для первого. Такие закономерности связаны с арифметическими свойствами нулей функций Бесселя.

Библиография: 26 названий.

DOI: $10.4213 / \mathrm{mzm} 10535$

1. Введение. Пусть $d \in \mathbb{N}, \mathbb{R}^{d}-d$-мерное действительное евклидово пространство со скалярным произведением $(x, y)$ и нормой $|x|=\sqrt{(x, x)}, \sigma>0, B_{\sigma}=\left\{x \in \mathbb{R}^{d}\right.$ : $|x| \leqslant \sigma\}-$ евклидов шар,

$$
v_{k}(x)=\prod_{\alpha \in R_{+}}|(\alpha, x)|^{2 k(\alpha)}
$$

- обобщенный степенной вес или вес Данкля, определяемый положительной подсистемой $R_{+}$системы корней $R \subset \mathbb{R}^{d}$ и функцией кратности $k(\alpha): R \rightarrow \mathbb{R}_{+}$, инвариантной относительно группы отражений $G(R)$, порожденной $R$,

$$
c_{k}=\int_{\mathbb{R}^{d}} e^{-|x|^{2} / 2} v_{k}(x) d x
$$

- интеграл Макдональда-Мета-Сельберга, $d \mu_{k}(x)=c_{k}^{-1} v_{k}(x) d x, L_{2, k}\left(\mathbb{R}^{d}\right)$ - гильбертово пространство комплексных измеримых по Лебегу на $\mathbb{R}^{d}$ функций $f$ с конечной нормой

$$
\|f\|_{2, k}=\left(\int_{\mathbb{R}^{d}}|f(x)|^{2} d \mu_{k}(x)\right)^{1 / 2} .
$$

При $k(\alpha) \equiv 0$ будем использовать обозначение $\|f\|_{2}$.

Работа выполнена при поддержке Российского фонда фундаментальных исследований (грант № 13-01-00045) и Министерства образования и науки РФ (госзадания №№ 5414ГЗ, 1.1333.2014K).

(С) В.И. Иванов, А. В. Иванов, 2014 
Гармонический анализ в $L_{2, k}\left(\mathbb{R}^{d}\right)$ осуществляется с помощью прямого и обратного преобразований Данкля

$$
\widehat{f}(y)=\int_{\mathbb{R}^{d}} f(x) \overline{e_{k}(x, y)} d \mu_{k}(x), \quad f(x)=\int_{\mathbb{R}^{d}} \widehat{f}(y) e_{k}(x, y) d \mu_{k}(y),
$$

где $e_{k}(x, y)$ - обобщенная экспонента, определяемая с помощью дифференциальноразностных операторов Данкля, многие свойства которой аналогичны свойствам экспоненты $e^{i(x, y)}$. Для преобразований Данкля выполнено равенство Парсеваля (см. [1]).

Пусть $C_{b}(X)$ - пространство непрерывных ограниченных функций на локально компактном множестве $X, \operatorname{supp} f-$ носитель функции $f$.

Для функции $f \in L_{2, k}\left(\mathbb{R}^{d}\right)$

$$
E_{\sigma}(f)_{2, k}=\inf \left\{\|f-g\|_{2, k}: g \in L_{2, k}\left(\mathbb{R}^{d}\right), \operatorname{supp} \widehat{g} \subset B_{\sigma}\right\}
$$

- величина наилучшего приближения целыми функциями экспоненциального сферического типа $\sigma>0$.

Пусть $Г(x)$ - гамма-функция Эйлера, $r \in \mathbb{N}$,

$$
\left(\begin{array}{l}
r \\
s
\end{array}\right)=\frac{\Gamma(r+1)}{\Gamma(s+1) \Gamma(r-s+1)}, \quad M_{r}=\left\{(-1)^{s}\left(\begin{array}{l}
r \\
s
\end{array}\right)\right\}_{s \in \mathbb{Z}} .
$$

Последовательность $M_{r}$ отлична от нуля только при $s=0,1, \ldots, r$.

Посредством свертки образуем новую последовательность

$$
\nu_{s}=(-1)^{s} \sum_{l \in \mathbb{Z}}\left(\begin{array}{c}
r \\
s+l
\end{array}\right)\left(\begin{array}{l}
r \\
l
\end{array}\right)=(-1)^{s}\left(\begin{array}{c}
2 r \\
r-s
\end{array}\right)
$$

Она отлична от нуля при $s=-r,-r+1, \ldots, r$. Определим функцию

$$
\varphi_{r}(t, y)=\sum_{s=-r}^{r} \nu_{s} e_{k}(s t, y), \quad t, y \in \mathbb{R}^{d}
$$

Используя интегральное представление обобщенной экспоненты [2]

$$
e_{k}(x, y)=\int_{\mathbb{R}^{d}} e^{i\langle\xi, y\rangle} d \mu_{k}^{x}(\xi)
$$

где $\mu_{k}^{x}$ - вероятностная борелевская мера, носитель которой лежит в выпуклой оболочке $\operatorname{co}\{g x: g \in G(R)\}$ орбиты $x$ относительно группы $G(R)$, получим

$$
\varphi_{r}(t, y)=\int_{\mathbb{R}^{d}} \sum_{s=-r}^{r} \nu_{s} e^{i s\langle\xi, y\rangle} d \mu_{k}^{t}(\xi)=\int_{\mathbb{R}^{d}}\left|\sum_{s=0}^{r}(-1)^{s}\left(\begin{array}{c}
r \\
s
\end{array}\right) e^{i s\langle\xi, y\rangle}\right|^{2} d \mu_{k}^{t}(\xi) \geqslant 0 .
$$

Для $\varphi_{r}(t, y)$ выполнены также свойства

$$
\varphi_{r}(t, y) \in C_{b}\left(\mathbb{R}^{d} \times \mathbb{R}^{d}\right), \quad \varphi_{r}(t, y)=\varphi_{r}(y, t), \quad \varphi_{r}(0, y)=0 .
$$


Перечисленные свойства $\varphi_{r}(t, y)$ позволяют для $f \in L_{2, k}\left(\mathbb{R}^{d}\right)$ определить модуль непрерывности порядка $r$ [3]:

$$
\omega_{r}(\tau, f)_{2, k}=\sup _{|t| \leqslant \tau}\left(\int_{\mathbb{R}^{d}} \varphi_{r}(t, y)|\widehat{f}(y)|^{2} d \mu_{k}(y)\right)^{1 / 2}, \quad \tau>0 .
$$

Если $v_{k}(x)=1(k(\alpha) \equiv 0)-$ единичный вес и

$$
\Delta_{t}^{r} f(x)=\sum_{s=0}^{r}(-1)^{s}\left(\begin{array}{l}
r \\
s
\end{array}\right) f(x+s t)
$$

- разностный оператор порядка $r$, то модуль непрерывности

$$
\omega_{r}(\tau, f)_{2, k}=\sup _{|t| \leqslant \tau}\left\|\Delta_{t}^{r} f(x)\right\|_{2}
$$

совпадает с обычным модулем непрерывности порядка $r$.

Константа Джексона-Стечкина

$$
D_{r}(\sigma, \tau)_{2, k}=\sup \left\{\frac{E_{\sigma}(f)_{2, k}}{\omega_{r}(\tau, f)_{2, k}}: f \in L_{2, k}\left(\mathbb{R}^{d}\right)\right\}
$$

есть наименьшая константа в неравенстве Джексона-Стечкина

$$
E_{\sigma}(f)_{2, k} \leqslant D \omega_{r}(\tau, f)_{2, k} .
$$

Для константы Джексона-Стечкина установлены следующие свойства:

1) $D_{r}(\sigma, \tau)_{2, k}=D_{r}(1, \sigma \tau)_{2, k}$, поэтому в дальнейшем можно считать, что $\sigma=1$ [4];

2) $D_{r}(1, \tau)_{2, k}$ как функция $\tau>0$ конечна и непрерывна [4];

3) для любого $\tau>0$ справедлива нижняя оценка [4]:

$$
D_{r}(1, \tau)_{2, k} \geqslant\left(\begin{array}{c}
2 r \\
r
\end{array}\right)^{-1 / 2}
$$

4) для произвольного веса Данкля (1.1), $r \in \mathbb{N}$, функции $f \in L_{2, k}\left(\mathbb{R}^{d}\right), \sigma>0$ и некоторого $\tau^{*}=\tau^{*}(d, k, r)$ справедливо точное неравенство Джексона [5]:

$$
E_{\sigma}(f)_{2, k} \leqslant\left(\begin{array}{c}
2 r \\
r
\end{array}\right)^{-1 / 2} \omega_{r}\left(\frac{\tau^{*}}{\sigma}, f\right)_{2, k}
$$

В случае единичного веса неравенство (1.2) доказано Васильевым [6]. Для этого случая Горбачев [7] получил эффективную оценку $\tau^{*} \leqslant 2 q_{d / 2}$. Здесь $q_{d / 2}$ - наименьший положительный нуль функции Бесселя $J_{d / 2}(x)$.

Наименьшее значение $\tau^{*}$ в неравенстве (1.2) называется оптимальным аргументом. По определению оптимальный аргумент

$$
\tau_{r, k}=\min \left\{\tau>0: D_{r}(1, \tau)_{2, k}=\left(\begin{array}{c}
2 r \\
r
\end{array}\right)^{-1 / 2}\right\} .
$$

Существование минимума вытекает из непрерывности и невозрастания константы Джексона-Стечкина при $\tau>0$ и ее неограниченности при $\tau \rightarrow 0+0$. 
Наиболее полно оптимальные аргументы исследованы только для первого модуля непрерывности. Первый оптимальный аргумент был найден в периодическом случае Черных [8], [9]. В $L_{2}\left(\mathbb{R}^{d}\right)$ оптимальные аргументы найдены Горбачевым [10]. Отметим также работу Бердышевой [11]. В $L_{2, k}\left(\mathbb{R}^{d}\right)$ оптимальные аргументы найдены в работах [12]-[14].

Пусть $v_{k}$ - произвольный вес Данкля (1.1),

$$
\lambda_{k}=\frac{d}{2}-1+\sum_{\alpha \in R_{+}} k(\alpha) .
$$

Мы доказываем следующие утверждения.

Tеорема 1.1. Если $\lambda_{k}=1 / 2$, то для всех $r \in \mathbb{N}$

$$
\tau_{r, k}=\tau_{1, k}=2 \pi .
$$

Teорема 1.2. Eсли $\lambda_{k} \neq 1 / 2$, mo

$$
\tau_{2, k}>\tau_{1, k}
$$

Отметим, что равенство $\lambda_{k}=1 / 2$ возможно только в размерностях $d=1,2,3$, причем при $d=3$ вес - единичный.

Равенство (1.4) в $L_{2}\left(\mathbb{R}^{3}\right)$ при $r=2$ доказано Горбачевым и Странковским [15]. Неравенство $(1.5)$ в $L_{2}(\mathbb{R})$ доказано Арестовым и Бабенко [16].

2. Редукция к одномерному неравенству Джексона-Стечкина. В [3] доказано, что константа Джексона-Стечкина в $L_{2, k}\left(\mathbb{R}^{d}\right)$ совпадает с константой Джексона-Стечкина в пространстве $L_{2}\left(\mathbb{R}_{+}\right)$со степенным весом.

Пусть $\lambda \geqslant-1 / 2, J_{\lambda}(x)$ - функция Бесселя порядка $\lambda, j_{\lambda}(x)=2^{\lambda} \Gamma(\lambda+1) J_{\lambda}(x) / x^{\lambda}-$ нормированная функция Бесселя, $b_{\lambda}=2^{\lambda} \Gamma(\lambda+1), L_{2, \lambda}\left(\mathbb{R}_{+}\right)$- пространство комплексных измеримых по Лебегу на $\mathbb{R}_{+}$функций $f$ с конечной нормой

$$
\|f\|_{2, \lambda}=\left(b_{\lambda}^{-1} \int_{0}^{\infty}|f(t)|^{2} t^{2 \lambda+1} d t\right)^{1 / 2}, \quad \tilde{f}(s)=b_{\lambda}^{-1} \int_{0}^{\infty} f(r) j_{\lambda}(r s) r^{2 \lambda+1} d r
$$

- преобразование Ганкеля (см. [17; гл. V]). Преобразование Ганкеля является сужением на четные функции преобразования Данкля в $L_{2, k}(\mathbb{R})$ при $k(\alpha)=\lambda+1 / 2$. Обратное преобразование Ганкеля совпадает с прямым.

Рассмотрим непрерывную на $\mathbb{R}_{+}$функцию

$$
\alpha_{r}(t)=\sum_{l \in \mathbb{Z}} \nu_{l} j_{\lambda}(l t) .
$$

Для нее выполнены условия [4]

$$
\alpha_{r}(t) \in C_{b}\left(\mathbb{R}_{+}\right), \quad \alpha_{r}(t) \geqslant 0, \quad \alpha_{r}(0)=0 .
$$

Пусть

$$
\begin{aligned}
E_{\sigma}(f)_{2, \lambda} & =\inf \left\{\|f-g\|_{2, \lambda}: g \in L_{2, \lambda}\left(\mathbb{R}_{+}\right), \operatorname{supp} \widetilde{g} \subset[0, \sigma]\right\} \\
& =\left(b_{\lambda}^{-1} \int_{\sigma}^{\infty}|\widetilde{f}(s)|^{2} s^{2 \lambda+1} d s\right)^{1 / 2}
\end{aligned}
$$


- величина наилучшего приближения функции $f \in L_{2, \lambda}\left(\mathbb{R}_{+}\right)$четными целыми функциями экспоненциального типа,

$$
\omega_{r}(\delta, f)_{2, \lambda}=\sup _{0 \leqslant t \leqslant \delta}\left(b_{\lambda}^{-1} \int_{0}^{\infty} \alpha_{r}(t s)|\tilde{f}(s)|^{2} s^{2 \lambda+1} d s\right)^{1 / 2}
$$

- ее модуль непрерывности порядка $r$,

$$
D_{r}(\sigma, \delta)_{2, \lambda}=\sup \left\{\frac{E_{\sigma}(f)_{2, \lambda}}{\omega_{r}(\delta, f)_{2, \lambda}}: f \in L_{2, \lambda}\left(\mathbb{R}_{+}\right)\right\}
$$

- константа Джексона-Стечкина в пространстве $L_{2, \lambda}\left(\mathbb{R}_{+}\right)$.

Теорема 2.1 [3]. Если $\lambda_{k}$ определено в (1.3), $r \in \mathbb{N}, \sigma, \tau>0$, то

$$
D_{r}(\sigma, \tau)_{2, k}=D_{r}(\sigma, \tau)_{2, \lambda_{k}} .
$$

Пусть $\tau_{r, \lambda}$ - оптимальный аргумент в $L_{2, \lambda}\left(\mathbb{R}_{+}\right)$, т.е.

$$
\tau_{r, \lambda}=\min \left\{\tau>0: D_{r}(1, \tau)_{2, \lambda}=\left(\begin{array}{c}
2 r \\
r
\end{array}\right)^{-1 / 2}\right\} .
$$

Согласно теореме 2.1 теоремы 1.1, 1.2 будут вытекать из следующих утверждений.

ТеОРема 2.2. Если $\lambda=1 / 2$, то для всех $r \in \mathbb{N}$

$$
\tau_{r, \lambda}=\tau_{1, \lambda}=2 \pi
$$

TEOPEMA 2.3. Eсли $\lambda \neq 1 / 2$, mo

$$
\tau_{2, \lambda}>\tau_{1, \lambda}
$$

Известно, что при $\lambda \geqslant-1 / 2 \tau_{1, \lambda}=2 q_{\lambda}$, где $q_{\lambda}$ - наименьший положительный нуль функции Бесселя $J_{\lambda}(x)[10]$, и $\tau_{2,1 / 2}=\tau_{1,1 / 2}=2 \pi$ [15]. В [18] получена оценка $\tau_{r, \lambda} \leqslant$ $2 q_{\lambda+1}$.

3. Связь с обобщенной задачей Логана. Пусть $S^{+}[0,1]-$ множество неубывающих на отрезке $[0,1]$ функций $\mu(x)$, для которых $\mu(1)-\mu(0)=1$,

$$
K_{\lambda}=\left\{f(x): f(x)=\int_{0}^{1} j_{\lambda}(t x) d \mu(t), \mu \in S^{+}[0,1]\right\}
$$

- класс четных положительно определенных целых функций экспоненциального типа $1, \theta(f)=\sup \{x>0: f(x)>0\}, a_{s}=\nu_{s} /\left|\nu_{1}\right|, s=1, \ldots, r$,

$$
F_{r}(x, f)=-\sum_{s=1}^{r} a_{s} f(s x)
$$

Отметим, что $a_{1}=-1$,

$$
\begin{gathered}
\left|a_{s}\right| \downarrow, \quad\left|a_{s}\right|<1, \quad \operatorname{sgn} a_{s}=(-1)^{s}, \quad 1-\sum_{k=2}^{s} a_{k} \in(0,1), \quad s=2, \ldots, r, \\
\sum_{s=2}^{r} \frac{\left|a_{s}\right|}{s^{2}}<\frac{2}{3} .
\end{gathered}
$$


Обобщенная задача Логана состоит в вычислении величины

$$
\Lambda_{r}\left(K_{\lambda}\right)=\inf \left\{\theta\left(F_{r}(f)\right): f \in K_{\lambda}\right\}
$$

Логан [19] поставил и решил эту задачу в случае $\lambda=-1 / 2, F_{1}(t, f)=f(t)$. Примеры решения задачи Логана можно найти в [10], [11], [13], [14].

Tеорема 3.1 [20], [15]. Eсли $r \in \mathbb{N}, \lambda \geqslant-1 / 2$, mo

$$
\tau_{r, \lambda}=\Lambda_{r}\left(K_{\lambda}\right)
$$

В дальнейшем будем исследовать обобщенную задачу Логана. В [20] доказано, что в обобщенной задаче Логана существует экстремальная функция $f \in K_{\lambda}$, для которой $\theta\left(F_{r}(f)\right)<\infty$ и $F_{r}(f) \in L_{1,1 / 2}\left(\mathbb{R}_{+}\right)$. Здесь $L_{1, \lambda}\left(\mathbb{R}_{+}\right)$- пространство комплексных измеримых по Лебегу на $\mathbb{R}_{+}$функций $f$ с конечной нормой

$$
\|f\|=\int_{0}^{\infty}|f(t)| t^{2 \lambda+1} d t .
$$

4. Обобщенная задача Логана при $\lambda=1 / 2$. Для получения верхней оценки рассмотрим функцию

$$
f(x)=\frac{j_{1 / 2}^{2}(x / 2)}{1-(x / 2 \pi)^{2}}=\left(\frac{\sin (x / 2)}{x / 2}\right)^{2} \frac{1}{1-(x / 2 \pi)^{2}} .
$$

Так как

$$
f(x)=4 \int_{0}^{1} j_{1 / 2}(x t) t \sin ^{2} \pi t d t
$$

то $f \in K_{1 / 2}$. Имеем

$$
F_{r}(x, f)=\frac{\sin ^{2}(x / 2)}{(x / 2)^{2}\left(1-(x / 2 \pi)^{2}\right)}\left\{1-\sum_{s=2}^{r} \frac{a_{s}}{s^{2}} \frac{\sin ^{2}(s x / 2)}{\sin ^{2}(x / 2)} \frac{\left((x / 2 \pi)^{2}-1\right)}{\left((s x / 2 \pi)^{2}-1\right)}\right\} .
$$

Пусть $s \geqslant 2$. Так как для $x \geqslant 2 \pi$

$$
0 \leqslant \frac{\sin ^{2}(s x / 2)}{\sin ^{2}(x / 2)} \leqslant s^{2}, \quad 0 \leqslant \frac{(x / 2 \pi)^{2}-1}{(s x / 2 \pi)^{2}-1} \leqslant \frac{1}{s^{2}},
$$

то согласно (3.2)

$$
1-\sum_{s=2}^{r} \frac{a_{s}}{s^{2}} \frac{\sin ^{2}(s x / 2)}{\sin ^{2}(x / 2)} \frac{\left((x / 2 \pi)^{2}-1\right)}{\left((s x / 2 \pi)^{2}-1\right)} \geqslant 1-\sum_{s=2}^{r} \frac{\left|a_{s}\right|}{s^{2}}>\frac{1}{3} .
$$

Поэтому для $x \geqslant 2 \pi$ выполнено $F_{r}(x, f) \leqslant 0$ и $\Lambda_{r}\left(K_{1 / 2}\right) \leqslant 2 \pi$.

Для получения нижней оценки нам понадобятся некоторые вспомогательные факты и утверждения.

Известно, что для $\alpha_{1}, \ldots, \alpha_{r} \in \mathbb{R},\left|\alpha_{1}\right|+\cdots+\left|\alpha_{r}\right|<1, s \in \mathbb{Z}_{+}$

$$
\sum_{s_{1}=0}^{\infty} \cdots \sum_{s_{r}=0}^{\infty} \frac{\left(s_{2}+\cdots+s_{r}+s\right) !}{s_{1} ! \cdots s_{r} !} \alpha_{1}^{s_{1}} \cdots \alpha_{r}^{s_{r}}=\frac{s !}{\left(1-\alpha_{1}-\cdots-\alpha_{r}\right)^{s+1}},
$$

причем ряд (4.1) сходится абсолютно. 
Если для последовательности $\lambda_{k} \geqslant 0$ выполнено $\lambda_{k+1}-\lambda_{k} \geqslant \delta>0$, то для четной целой функции $g \in L\left(\mathbb{R}_{+}\right)$экспоненциального типа не выше $\tau>0$ справедливо неравенство Планшереля-Полиа [21]

$$
\sum_{k=1}^{\infty}\left|g\left(\lambda_{k}\right)\right| \leqslant \frac{4}{\pi \delta} e^{\tau \delta / 2} \int_{0}^{\infty}|g(x)| d x
$$

Отсюда для четной целой функции $g \in L_{1,1 / 2}\left(\mathbb{R}_{+}\right)$экспоненциального типа не выше 1 и произвольных $x>0$ вытекает неравенство

$$
\sum_{k=1}^{\infty} k^{2}|g(k x)| \leqslant \frac{c_{\tau}(x)}{x^{2}} \int_{0}^{\infty}|g(x)| x^{2} d x=\frac{c_{\tau}(x)}{x^{2}}\|g\|, \quad c_{\tau}(x)=2 \max \left(2 x^{-1}, \tau\right) .
$$

Для четной целой функции $g \in L_{1,1 / 2}\left(\mathbb{R}_{+}\right)$экспоненциального типа не выше 1 справедлива квадратурная формула [22], [23]

$$
\int_{0}^{\infty} g(x) x^{2} d x=(2 \pi)^{3} \sum_{k=1}^{\infty} k^{2} g(2 \pi k),
$$

причем ряд (4.3) сходится абсолютно.

Лемма 4.1. Если $r \in \mathbb{N}, f \in K_{1 / 2}, \theta\left(F_{r}(f)\right)<\infty$, mo $f \in L_{1,1 / 2}\left(\mathbb{R}_{+}\right), \tilde{f}(x) \geqslant 0$, $\operatorname{supp} \tilde{f} \subset[0,1]$ и для всех $x \in \mathbb{R}_{+}$

$$
f(x)=\sum_{s_{2}=0}^{\infty} \cdots \sum_{s_{r}=0}^{\infty} \frac{\left(s_{2}+\cdots+s_{r}\right) !}{s_{2} ! \cdots s_{r} !} a_{2}^{s_{2}} \cdots a_{r}^{s_{r}} F_{r}\left(2^{s_{2}} \cdots r^{s_{r}} x, f\right) .
$$

Ряд (4.4) сходится абсолютно при $x>0$ и равномерно при $x \geqslant \delta>0$, а также сходится в $L_{1,1 / 2}\left(\mathbb{R}_{+}\right)$.

ДоКАЗАТЕЛЬСТво. Положим $F(x)=F_{r}(x, f)$. При выполнении условий леммы $F(x) \in L_{1,1 / 2}\left(\mathbb{R}_{+}\right)[20]$.

При $x=0$ имеем $F(0)=\left(1-\sum_{s=2}^{r} a_{s}\right) f(0)$ и согласно (3.2), (4.1) ряд (4.4), рассматриваемый как повторный в порядке возрастания индекса, сходится к $f(0)$ :

$$
\sum_{s_{2}=0}^{\infty} \cdots \sum_{s_{r}=0}^{\infty} \frac{\left(s_{2}+\cdots+s_{r}\right) !}{s_{2} ! \cdots s_{r} !} a_{2}^{s_{2}} \cdots a_{r}^{s_{r}} F(0)=\left(1-\sum_{s=2}^{r} a_{s}\right)^{-1} F(0)=f(0) .
$$

Согласно (3.2), (4.1) и (4.2) при $\tau=r$ ряд (4.4) сходится абсолютно при $x>0$ (равномерно при $x \geqslant \delta>0$ ):

$$
\begin{aligned}
& \sum_{s_{2}=0}^{\infty} \cdots \sum_{s_{r}=0}^{\infty} \frac{\left(s_{2}+\cdots+s_{r}\right) !}{s_{2} ! \cdots s_{r} !}\left|a_{2}\right|^{s_{2}} \cdots\left|a_{r}\right|^{s_{r}}\left|F\left(2^{s_{2}} \cdots r^{s_{r}} x\right)\right| \\
& \quad \leqslant \frac{c_{r}(x)}{x^{2}} \sum_{s_{2}=0}^{\infty} \cdots \sum_{s_{r}=0}^{\infty} \frac{\left(s_{2}+\cdots+s_{r}\right) !}{s_{2} ! \cdots s_{r} !}\left(\frac{\left|a_{2}\right|}{2^{2}}\right)^{s_{2}} \cdots\left(\frac{\left|a_{r}\right|}{r^{2}}\right)^{s_{r}}\|F\| \leqslant \frac{3 c_{r}(x)}{x^{2}}\|F\| .
\end{aligned}
$$

Согласно (3.1) справедливо равенство

$$
f(x)=F(x)+\sum_{s=2}^{r} a_{s} f(s x) .
$$


Индукцией по $N$ выводим

$$
\begin{aligned}
f(x)= & \sum_{s=0}^{N-1} \sum_{s_{2}+\cdots+s_{r}=s} \frac{s !}{s_{2} ! \cdots s_{r} !} a_{2}^{s_{2}} \cdots a_{r}^{s_{r}} F\left(2^{s_{2}} \cdots r^{s_{r}} x\right) \\
& \quad+\sum_{s_{2}+\cdots+s_{r}=N} \frac{N !}{s_{2} ! \cdots s_{r} !} a_{2}^{s_{2}} \cdots a_{r}^{s_{r}} f\left(2^{s_{2}} \cdots r^{s_{r}} x\right) \\
= & \sum_{s=0}^{N-1} A_{s}(x)+B_{N}(x) .
\end{aligned}
$$

Ряд $\sum_{s=0}^{\infty} A_{s}(x)$ является группировкой ряда (4.4), поэтому он сходится абсолютно при $x>0$. Так как

$$
\int_{\mathbb{R}_{+}}\left|F\left(2^{s_{2}} \cdots r^{s_{r}} x\right)\right| x^{2} d x=\left(2^{s_{2}} \cdots r^{s_{r}}\right)^{-3} \int_{\mathbb{R}_{+}}|F(x)| x^{2} d x=\left(2^{s_{2}} \cdots r^{s_{r}}\right)^{-3}\|F\|,
$$

согласно (4.1) он сходится в $L_{1,1 / 2}\left(\mathbb{R}_{+}\right)$:

$$
\sum_{s=0}^{\infty}\left\|A_{s}\right\| \leqslant \sum_{s=0}^{\infty} \sum_{s_{2}+\cdots+s_{r}=s} \frac{s !}{s_{2} ! \cdots s_{r} !}\left(\frac{\left|a_{2}\right|}{2^{2}}\right)^{s_{2}} \cdots\left(\frac{\left|a_{r}\right|}{r^{2}}\right)^{s_{r}}\|F\| \leqslant 3\|F\| .
$$

Аналогично доказывается сходимость в $L_{1,1 / 2}\left(\mathbb{R}_{+}\right)$ряда (4.4).

Последовательность $B_{N}(x)$ фундаментальна в $L_{1,1 / 2}\left(\mathbb{R}_{+}\right)$, поэтому $f \in L_{1,1 / 2}\left(\mathbb{R}_{+}\right)$. Согласно (3.2) и (4.2) при $\tau=1$ для $x>0$ и $N \rightarrow \infty$

$$
\begin{aligned}
\left|B_{N}(x)\right| & =\left|\sum_{s_{2}+\cdots s_{r}=N} \frac{N !}{s_{2} ! \cdots s_{r} !} a_{2}^{s_{2}} \cdots a_{r}^{s_{r}} f\left(2^{s_{2}} \cdots r^{s_{r}} x\right)\right| \\
& \leqslant \frac{c_{1}(x)}{x^{2}}\|f\| \sum_{s_{2}+\cdots s_{r}=N} \frac{N !}{s_{2} ! \cdots s_{r} !}\left(\frac{\left|a_{2}\right|}{2^{2}}\right)^{s_{2}} \cdots\left(\frac{\left|a_{r}\right|}{r^{2}}\right)^{s_{r}} \leqslant \frac{c_{1}(x)}{x^{2}}\|f\|\left(\frac{2}{3}\right)^{N} \rightarrow 0 .
\end{aligned}
$$

Таким образом, ряд (4.4) для всех $x>0$ абсолютно сходится к $f(x)$.

По теореме Пэли-Винера [24] $\operatorname{supp} \tilde{f} \subset[0,1]$ и для всех $x \geqslant 0$

$$
f(x)=\sqrt{\frac{2}{\pi}} \int_{0}^{1} \tilde{f}(t) j_{1 / 2}(x t) d t=\int_{0}^{1} j_{1 / 2}(x t) d \mu(t),
$$

поэтому по теореме о единственности меры (см., например, [13]) $d \mu(t)=\sqrt{2 / \pi} \tilde{f}(t) d t$ и $\tilde{f}(t) \geqslant 0$. Лемма 4.1 доказана.

ЛЕмма 4.2. Если $r \in \mathbb{N}, f \in L_{1,1 / 2}\left(\mathbb{R}_{+}\right)$- четная целая функиия экспоненииального типа не выше 1 , mо

$$
\int_{0}^{\infty} F_{r}(x, f) x^{2} d x=\sum_{n=1}^{\infty} \rho_{n} F_{r}(2 \pi n, f),
$$

причем ряд (4.6) сходится абсолютно и для $n \in \mathbb{N}$

$$
0<c_{1}(r) n^{2} \leqslant \rho_{n} \leqslant c_{2}(r) n^{2} .
$$


ДокАзАтЕЛЬСтво. Согласно (3.1), (3.2), (4.3), (4.4)

$$
\begin{aligned}
\int_{0}^{\infty} & F_{r}(x, f) x^{2} d x \\
& =-\sum_{s=1}^{r} a_{s} \int_{0}^{\infty} f(s x) x^{2} d x=-\sum_{s=1}^{r} \frac{a_{s}}{s^{3}} \int_{0}^{\infty} f(x) x^{2} d x \\
& =a(r) \sum_{k=1}^{\infty} k^{2} \sum_{s_{2}=0}^{\infty} \cdots \sum_{s_{r}=0}^{\infty} \frac{\left(s_{2}+\cdots+s_{r}\right) !}{s_{2} ! \cdots s_{r} !} a_{2}^{s_{2}} \cdots a_{r}^{s_{r}} F_{r}\left(2 \pi k 2^{s_{2}} \cdots r^{s_{r}}, f\right) \\
& =a(r) \sum_{n=1}^{\infty} n^{2} F_{r}(2 \pi n, f) \quad \sum_{k 2^{s_{2}} \cdots r^{s_{r}}=n} \frac{\left(s_{2}+\cdots+s_{r}\right) !}{s_{2} ! \cdots s_{r} !}\left(\frac{a_{2}}{2^{2}}\right)^{s_{2}} \cdots\left(\frac{a_{r}}{r^{2}}\right)^{s_{r}} \\
& =\sum_{n=1}^{\infty} \rho_{n} F_{r}(2 \pi n, f), \quad a(r)=-(2 \pi)^{3} \sum_{s=1}^{r} \frac{a_{s}}{s^{3}}>0 .
\end{aligned}
$$

Положим

$$
J_{n}=\sum_{k 2^{s_{2} \ldots r^{s_{r}}=n}} \frac{\left(s_{2}+\cdots+s_{r}\right) !}{s_{2} ! \cdots s_{r} !}\left(\frac{a_{2}}{2^{2}}\right)^{s_{2}} \cdots\left(\frac{a_{r}}{r^{2}}\right)^{s_{r}} .
$$

Отметим, что представление $k 2^{s_{2}} \cdots r^{s_{r}}=n$ верно при $k=n, s_{2}=\cdots=s_{r}=0$.

Пусть сначала $r=2$. Тогда $a_{2}=1 / 4$,

$$
1 \leqslant J_{n}=\sum_{k 2^{s}=n}\left(\frac{a_{2}}{4}\right)^{s} \leqslant \sum_{s=0}^{\infty} 16^{-s} \leqslant 2
$$

и $a(r) n^{2} \leqslant \rho_{n}=a(r) J_{n} n^{2} \leqslant 2 a(r) n^{2}$.

Далее считаем $r \geqslant 3$. Так как в силу (3.2), (4.1)

$$
J_{n} \leqslant \sum_{s_{2}=0}^{\infty} \cdots \sum_{s_{r}=0}^{\infty} \frac{\left(s_{2}+\cdots+s_{r}\right) !}{s_{2} ! \cdots s_{r} !}\left(\frac{\left|a_{2}\right|}{2^{2}}\right)^{s_{2}} \cdots\left(\frac{\left|a_{r}\right|}{r^{2}}\right)^{s_{r}} \leqslant\left(1-\sum_{s=2}^{r} \frac{\left|a_{s}\right|}{s^{2}}\right)^{-1} \leqslant 3,
$$

$F_{r}(x, f) \in L_{1,1 / 2}\left(\mathbb{R}_{+}\right)$, то ряд (4.7) сходится абсолютно и $\rho_{n} \leqslant 3 a(r) n^{2}$.

Пусть $t=[(r-1) / 2], \tau=[r / 2]$,

$$
\sigma_{1}=\sum_{s=1}^{\tau} \frac{\left|a_{2 s}\right|}{(2 s)^{2}}, \quad \sigma_{2}=\sum_{s=1}^{t} \frac{\left|a_{2 s+1}\right|}{(2 s+1)^{2}} .
$$

Справедливы оценки

$$
\sigma_{1} \leqslant \sum_{s=1}^{\infty} \frac{1}{(2 s)^{2}}=\frac{\pi^{2}}{24}, \quad \sigma_{2} \leqslant \sum_{s=1}^{\infty} \frac{1}{(2 s+1)^{2}}=\frac{\pi^{2}}{8}-1 .
$$

Так как $a_{2 j}>0, j=1, \ldots, \tau, a_{2 i+1}<0, i=1, \cdots, t$, то применяя (4.1), (4.8), получим

$$
\begin{aligned}
J_{n} \geqslant 1-\sum_{l=0}^{\infty} & \sum_{s_{3}+\cdots+s_{2 t+1}=2 l+1} \frac{1}{s_{3} ! \cdots s_{2 t+1} !}\left(\frac{\left|a_{3}\right|}{3^{2}}\right)^{s_{3}} \cdots\left(\frac{\left|a_{2 t+1}\right|}{(2 t+1)^{2}}\right)^{s_{2 t+1}} \\
& \times \sum_{s_{2}=0}^{\infty} \cdots \sum_{s_{2 \tau}=0}^{\infty} \frac{\left(s_{2}+\cdots+s_{2 \tau}+2 l+1\right) !}{s_{2} ! \cdots s_{2 \tau} !}\left(\frac{a_{2}}{2^{2}}\right)^{s_{2}} \cdots\left(\frac{a_{2 \tau}}{(2 \tau)^{2}}\right)^{s_{2 \tau}}
\end{aligned}
$$




$$
\begin{aligned}
& =1-\sum_{l=0}^{\infty} \frac{1}{\left(1-\sigma_{1}\right)^{2 l+2}} \sum_{s_{3}+\cdots+s_{2 t+1}=2 l+1} \frac{(2 l+1) !}{s_{3} ! \cdots s_{2 t+1} !}\left(\frac{\left|a_{3}\right|}{3^{2}}\right)^{s_{3}} \cdots\left(\frac{\left|a_{2 t+1}\right|}{(2 t+1)^{2}}\right)^{s_{2 t+1}} \\
& =1-\frac{1}{1-\sigma_{1}} \sum_{l=0}^{\infty}\left(\frac{\sigma_{2}}{1-\sigma_{1}}\right)^{2 l+1}=1-\frac{\sigma_{2}}{\left(1-\sigma_{1}\right)^{2}-\sigma_{2}^{2}} \geqslant 1-\frac{3 / 2-12 / \pi^{2}}{2-\pi^{2} / 6} \geqslant 0.1 .
\end{aligned}
$$

Таким образом, при $r \geqslant 3$ выполнено $\rho_{n} \geqslant 0,1 a(r) n^{2}$. Лемма 4.2 доказана.

Для функции $f_{\theta}(x)=f(\theta x), \theta \in(0,1], F_{r}\left(x, f_{\theta}\right)=F_{r}(\theta x, f)$. Применяя лемму 4.2 , получим следующее утверждение.

ЛЕмма 4.3. Если $r \in \mathbb{N}, f \in L_{1,1 / 2}\left(\mathbb{R}_{+}\right)$- четная челая функиия экспоненииального типа не выше $1, \theta \in(0,1]$, то

$$
\int_{0}^{\infty} F_{r}(x, f) x^{2} d x=\theta^{3} \sum_{n=1}^{\infty} \rho_{n} F_{r}(2 \pi \theta n, f) .
$$

Теперь мы можем в обобщенной задаче Логана получить нижнюю оценку. Пусть для некоторых $\epsilon>0$ и функции $f \in K_{1 / 2} \cap L_{1,1 / 2}\left(\mathbb{R}_{+}\right)$

$$
f(x) \leqslant 0, \quad x \geqslant(1-\epsilon) 2 \pi .
$$

Так как по лемме $4.1 \tilde{f}(0) \geqslant 0$, применяя $(4.9),(4.10)$, для $\theta \in[1-\epsilon, 1]$ получим

$$
0 \leqslant-\sum_{s=1}^{r} \frac{a_{s}}{s^{3}} \int_{0}^{\infty} f(x) x^{2} d x=\int_{0}^{\infty} F_{r}(x, f) x^{2} d x=\theta^{3} \sum_{n=1}^{\infty} \rho_{n} F_{r}(2 \pi \theta n, f) \leqslant 0 .
$$

Отсюда для $\theta \in[1-\epsilon, 1] \quad F_{r}(2 \pi \theta, f)=0$, а по лемме 4.1 и $f(2 \pi \theta)=0$. Из аналитичности $f$ вытекает, что $f \equiv 0$, что противоречит условию $f(0)=1$. Значит, $\Lambda_{r}\left(K_{1 / 2}\right) \geqslant 2 \pi$. Нижняя оценка получена.

Итак, нами доказана следующая теорема.

TEOPEMA 4.4. Eсли $r \in \mathbb{N}$, mo

$$
\Lambda_{r}\left(K_{1 / 2}\right)=2 \pi
$$

Из теорем 3.1, 4.4 вытекают теоремы 1.1, 2.2.

5. Обобщенная задача Логана при $\lambda \neq 1 / 2$. Пусть $\lambda \geqslant-1 / 2, q_{\lambda, 1}<q_{\lambda, 2}<$ $\cdots<q_{\lambda, s}<\cdots-$ положительные нули функции Бесселя $J_{\lambda}(x)$,

$$
E_{N}=\min \left\{\left|2 q_{\lambda, k}-q_{\lambda, s}\right|: k, s \geqslant N\right\}
$$

Известна следующая асимптотика нулей функции Бесселя [25; с. 557]:

$$
q_{\lambda, s}=\beta-\frac{4 \lambda^{2}-1}{8 \beta}+O\left(\frac{1}{s^{3}}\right), \quad \beta=\left(s+\frac{1}{2} \lambda-\frac{1}{4}\right) \pi .
$$

Лемма 5.1. Если $\lambda \neq 1 / 2$, то для некоторого $N \in \mathbb{N}$

$$
\left\{q_{\lambda, s}\right\}_{s=N}^{\infty} \cap\left\{2 q_{\lambda, s}\right\}_{s=N}^{\infty}=\varnothing .
$$


ДоказАТЕЛЬство. Свойство (5.2) эквивалентно неравенству $E_{N}>0$ для некоторого $N \in \mathbb{N}$.

Если $\lambda \neq 2 m+1 / 2, m \in \mathbb{Z}_{+}$, то $\lambda / 2-1 / 4$ не является целым и согласно $(5.1)$

$$
2 q_{\lambda, k}-q_{\lambda, s}=2 k-s+\frac{1}{2} \lambda-\frac{1}{4}+O\left(\frac{1}{k}\right)+O\left(\frac{1}{s}\right),
$$

поэтому $E_{N}>0$ для некоторого $N \in \mathbb{N}$.

Если $\lambda=2 m+1 / 2, m \in \mathbb{Z}_{+}$, то согласно (5.1)

$$
2 q_{\lambda, k}-q_{\lambda, s}=2 k-s+m-\frac{\left(4 \lambda^{2}-1\right)(2 s-k+m)}{8 \pi(k+m)(s+m)}+O\left(\frac{1}{k^{3}}\right)+O\left(\frac{1}{s^{3}}\right) .
$$

Разбирая случаи $2 k-s+m \neq 0$ и $2 k-s+m=0$, опять приходим к заключению, что $E_{N}>0$ для некоторого $N \in \mathbb{N}$. Лемма 5.1 доказана.

Рассмотрим обобщенную задачу Логана для функций $f \in K_{\lambda}$ при $\lambda \neq 1 / 2, r=2$, $F_{2}(x, f)=f(x)-f(2 x) / 4$. Из $(4.5)$

$$
f(x)=\sum_{s=0}^{N-1} 4^{-s} F_{2}\left(2^{s} x, f\right)+4^{-N} f\left(2^{N} x\right),
$$

поэтому для всех $x \geqslant 0$

$$
f(x)=\sum_{s=0}^{\infty} 4^{-s} F_{2}\left(2^{s} x, f\right),
$$

причем ряд сходится абсолютно, равномерно и в $L_{1, \lambda}\left(\mathbb{R}_{+}\right)$. Впервые равенство (5.3) появилось в [15]. Из него вытекает, что $\theta(f) \leqslant \theta\left(F_{2}(f)\right)$. Если $\theta\left(F_{2}(f)\right)=2 q_{\lambda, 1}=2 q_{\lambda}$, то $\theta(f) \leqslant 2 q_{\lambda}$. Единственной такой функцией $f^{*} \in K_{\lambda}[26 ;$ с. 110$]$ является функция

$$
f^{*}(x)=\frac{j_{\lambda}^{2}(x / 2)}{1-\left(x / 2 q_{\lambda}\right)^{2}} .
$$

Согласно (5.2) для нее $\theta\left(F_{2}\left(f^{*}\right)\right)=\infty$. Следовательно, для любой функции $f \in K_{\lambda}$ $\theta\left(F_{2}(f)\right)>2 q_{\lambda}$, поэтому при $\lambda \neq 1 / 2 \Lambda_{2}\left(K_{\lambda}\right)>2 q_{\lambda}=\Lambda_{1}\left(K_{\lambda}\right)$.

Итак, нами доказана

TеОрема 5.2. Если $\lambda \neq 1 / 2$, mo

$$
\Lambda_{2}\left(K_{\lambda}\right)>\Lambda_{1}\left(K_{\lambda}\right) .
$$

Из теорем 3.1, 5.2 вытекают теоремы 1.2, 2.3.

6. Заключение. Почему в обобщенной задаче Логана различаются случаи $\lambda=$ $1 / 2$ и $\lambda \neq 1 / 2$ ? Это связано с арифметическими свойствами нулей функций Бесселя. При $\lambda=1 / 2 \quad q_{\lambda, s}=\pi s$ и для любого $k \in \mathbb{N}$

$$
\left\{k q_{\lambda, s}\right\}_{s=1}^{\infty} \subset\left\{q_{\lambda, s}\right\}_{s=1}^{\infty} .
$$

На этом свойстве нулей функции $J_{1 / 2}(x)$ основано доказательство теоремы 4.4. Для $\lambda \neq 1 / 2$ доказательство теоремы 5.2 основано на свойстве нулей (5.2).

Мы предполагаем, что для нулей функций Бесселя справедливы более сильные утверждения. 
ГиПОТЕЗА 1. При $\lambda \neq 1 / 2$

$$
\left\{q_{\lambda, s}\right\}_{s=1}^{\infty} \cap\left\{2 q_{\lambda, s}\right\}_{s=1}^{\infty}=\varnothing .
$$

ГиПотезА 2. При $\lambda \neq \pm 1 / 2$ для любъх $s \neq l \quad q_{\lambda, s} / q_{\lambda, l} \notin \mathbb{Q}$.

\section{СПИСОК ЦИТИРОВАННОЙ ЛИТЕРАТУРЫ}

[1] M. Rösler, "Dunkl operators. Theory and applications", Orthogonal Polynomials and Special Functions, Lecture Notes in Math., 1817, Springer-Verlag, Berlin, 2002, 93-135.

[2] M. Rösler, "A positive radial product formula for the Dunkl kernel", Trans. Amer. Math. Soc., 355:6 (2003), 2413-2438.

[3] Ха Тхи Минь Хуе, "О связи многомерных и одномерных констант Джексона в пространствах $L_{2}$ со степенными весами”, Изв. ТулГУ. Естественные науки, 2012, №6, $114-123$.

[4] А. В. Иванов, В.И. Иванов, Ха Тхи Минь Хуе, "Обобщенная константа Джексона в пространстве $L_{2}\left(\mathbb{R}^{d}\right)$ с весом Данкля", Изв. ТулГУ. Естественные науки, 2013, № 3, 74-90.

[5] В. И. Иванов, Ха Тхи Минь Хуе, "Обобщенное неравенство Джексона в пространстве $L_{2}\left(\mathbb{R}^{d}\right)$ с весом Данкля", Тр. ИММ УрО РАН, 20, 2014, 109-118.

[6] С. Н. Васильев, "Неравенство Джексона в $L_{2}\left(\mathbb{R}^{N}\right)$ с обобщенным модулем непрерывности", Тр. ИММ УрО РАН, 16, 2010, 93-99.

[7] Д. В. Горбачев, "Оценка оптимального аргумента в точном многомерном $L_{2}$-неравенстве Джексона-Стечкина", Тр. ИММ УрО РАН, 20, 2014, 83-91.

[8] Н. И. Черных, "О неравенстве Джексона в $L_{2}$ ", Приближение функиий в среднем, Тр. МИАН СССР, 88, 1967, 71-74.

[9] V. V. Arestov, N. I. Chernykh, "On the $L_{2}$-approximation of periodic functions by trigonometric polynomials", Approximation and Functions Spaces, North-Holland, Amsterdam, 1981, 25-43.

[10] Д. В. Горбачев, “Экстремальные задачи для целых функций экспоненциального сферического типа", Матем. заметки, 68:2 (2000), 179-187.

[11] Е.Е. Бердышева, “Две взаимосвязанные экстремальные задачи для целых функций многих переменных", Матем. заметки, 66:3 (1999), 336-350.

[12] А. В. Иванов, "Некоторые экстремальные задачи для целых функций в весовых пространствах", Изв. ТулГУ. Естественные науки, 2010, № 1, 26-44.

[13] А. В. Иванов, “Задача Логана для целых функций многих переменных и константы Джексона в весовых пространствах", Изв. ТулГУ. Естественные науки, 2011, № 2, $29-58$.

[14] А. В. Иванов, В. И. Иванов, "Оптимальные аргументы в неравенстве Джексона в пространстве $L_{2}\left(\mathbb{R}^{d}\right)$ со степенным весом", Матем. заметки, 94:3 (2013), 338-348.

[15] Д. В. Горбачев, С. А. Странковский, "Одна экстремальная задача для четных положительно определенных целых функций экспоненциального типа", Матем. заметки, 80:5 (2006), 712-717.

[16] V. V. Arestov, A. G. Babenko, "On the optimal point in Jackson's inequality in $L_{2}(-\infty, \infty)$ with the second modulus of continuity", East J. Approx., 10:1-2 (2004), 201-214.

[17] Б. М. Левитан, И. С. Саргсян, Введение в спектральную теорию. Самосопряжсеннье обыкновенные дифференциальные операторы, Наука, М., 1970.

[18] Ха Тхи Минь Хуе, "Обобщенное неравенство Джексона-Стечкина в пространстве $L_{2}\left(\mathbb{R}^{d}\right)$ с весом Данкля", Изв. ТулГУ. Естественные науки, 2014, № 1, 63-82.

[19] B. F. Logan, "Extremal problems for positive-definite bandlimited functions. I. Eventually positive functions with zero integral", SIAM J. Math. Anal., 14:2 (1983), 249-253. 
[20] А. В. Иванов, В.И. Иванов, "Оптимальный аргумент в обобщенном неравенстве Джексона в пространстве $L_{2}\left(\mathbb{R}^{d}\right)$ с весом Данкля и обобщенная задача Логана", Изв. ТулГУ. Естественные науки, 2014, № 1, 22-36.

[21] M. Plancherel, G. Pólya, "Fonctions entiéres et intégrales de Fourier multiples", Comment. Math. Helv., 9 (1937), 224-248.

[22] C. Frappier, P. Oliver, "A quadrature formula involving zeros of Bessel functions", Math. Comp., 60:201 (1993), 303-316.

[23] G. R. Grozev, Q.I. Rahman, "A quadrature formulae with zeros of Bessel functions as nodes", Math. Comp., 64:210 (1995), 715-725.

[24] M. de Jeu, "Paley-Wiener theorems for the Dunkl transform", Trans. Amer. Math. Soc., 358:10 (2006), 4225-4250.

[25] Г.Н. Ватсон, Теория бесселевых функиий, Ч. 1, ИЛ, М., 1949.

[26] Д. В. Горбачев, Экстремальные задачи теории функиий и теории приближений и ux приложения, Дис. ... докт. физ.-матем. наук, Екатеринбург, 2006.

В. И. Иванов

Тульский государственный университет

E-mail: ivaleryi@mail.ru

А. В. Иванов

Тульский государственный университет

E-mail: d_bringer@mail.ru
Поступило 16.06.2014 